\title{
GIANT Magnetoimpedance Sensors
}

M agnetoinductance and giant magnetoimpedance (GMI) sensors have greatly benefited from the development of amorphous wires. These soft ferromagnetic substances exhibit exquisite sensitivity (in the nT range) and wide bandwidth $(\mathrm{MHz})$ in thin film structures. Combining these properties with surface wave technology produces passive, wireless sensors.

\section{Magnetoinductance}

Magnetoinductive effects in ferromagnetic conductors can be used for various sensors. Hans Christian Orsted of Denmark discovered the principles of magnetoinductance in 1820 . He found that whenever electricity flows through a wire, a magnetic field is produced around the wire. This produces magnetization in the conductor called magnetoinductance. If the current varies with time, then the magnetic flux in the conductor also varies and induces an electromotive force between the ends of the conductor that is superimposed on the ohmic voltage.

In a wire with a circular cross section, the circumferential magnetic field, $H$, induced by a constant current with the density, $j$, is $H=j r / 2$, where $r$ is the distance from the wire axis. For a wire with a $1-\mathrm{mm}$ diameter and a current density of $10^{6} \mathrm{~A} / \mathrm{m}^{2}$ (which is low enough to not increase the temperature by joule heating), the maximum magnitude of a magnetic field on the wire surface is $250 \mathrm{~A} / \mathrm{m}$. The circumferential reversal of the conductor magnetization must be of this order or lower to detect the magnetoinductive voltage easily against the ohmic

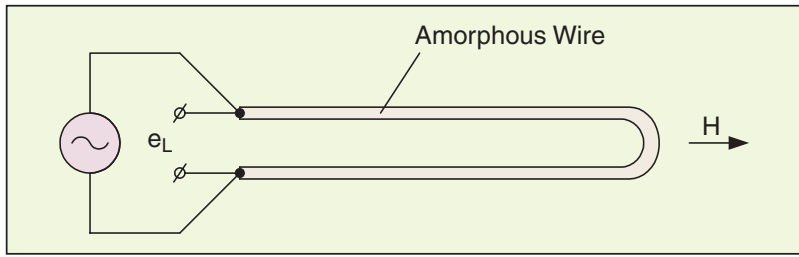

Fig. 1. Simple magnetoinductive head using an amorphous wire. (Reprinted with permission from [4].) background signal. Therefore, these applications require soft magnetic metals with high circumferential permeability.

The systematic study of magnetoinductive effects in soft magnetic conductors began with the development of amorphous wires. A large magnetoinductive effect was found in the zero-magnetostrictive, amorphous $\mathrm{CoFeSiB}$ wire that has a circumferential, bamboo-like domain structure in the outer shell. When an ac current of $1 \mathrm{kHz}$ conducts in a CoFeSiB wire, sharp peaks (about $0.2 \mathrm{~V}$ ) are induced on the background ohmic signal by the circumferential magnetization reversal in the outer shell. The peak amplitude decreases with an increasing external dc magnetic field. Using this effect, a simple magnetic head (Fig. 1) was constructed and used as a noncontact rotary encoder and a cordless data tablet.

\section{Giant Magnetoimpedance}

Another magnetoinductive effect observed in soft ferromagnetic metals is GMI. The ac impedance in GMI has a strong dependence on the applied magnetic field (Fig. 2). This effect occurs at high frequencies and can be explained by classical electrodynamics.

Radio frequency (RF) current is not homogeneous over the cross section of a conductor; it tends to concentrate near the conductor's surface and is called the skin effect. The exponential decay of current density from the surface towards the interior of the conductor is described by the skin depth: $\delta=\sqrt{2 \rho / \omega \mu}$. It depends on the circular frequency of the RF current, $\omega$, the resistivity $\rho$, and the permeability $\mu$. In nonferromagnetic metals, $\mu$ is independent of frequency and the applied magnetic field; its value is close to the permeability of a vacuum $\mu_{0}$. In ferromagnetic materials, however, the permeability depends on the frequency, the amplitude of the ac magnetic field, the magnitude and orientation of a bias dc magnetic field, mechanical strain, and temperature. The high permeability of soft magnetic metals and their strong dependance on the bias magnetic field are the origin of the GMI effect. 
The complex impedance $Z(\omega)=R+i X$ of a uniform conductor (Fig. 3 ) is the ratio of the voltage amplitude, $U$, to the amplitude of a sinusoidal current $I \sin \omega t$ passing through it. For a ferromagnetic wire with radius $a$ and length $l$ and for $\delta<<$ :

$$
Z=R_{\mathrm{dc}} \frac{a}{2 \delta}+i \omega L_{i} \frac{2 \delta}{a}=(1+i) a R_{\mathrm{dc}} \sqrt{\frac{\omega \mu}{2 \rho}} .
$$

The impedance equals the dc resistance for a uniform current density. This equation is valid only for linear elements when the voltage, $U$, is proportional to the current, $I$. A ferromagnetic conductor, however, is nonlinear. Consequently, the voltage is not proportional to the current; moreover, it contains harmonics of the basic frequency. Therefore, the term impedance should be used with care.

The impedance can be calculated if the current density, $j(r)$, in the conductor is known. If the relationship between $B$ and $H$ for a given conductor is known, then solving the Maxwell equations can generally give the current density.

At frequencies above $1 \mathrm{MHz}$, eddy currents heavily damp the domain wall movements, and only magnetization rotations are responsible for magnetic permeability. The minimum skin depth is $\delta_{m}=\sqrt{\alpha\left(\rho / \mu_{0} M_{s}\right)}$ which is, for soft magnetic amorphous alloys, about $0.1 \mu \mathrm{m}$. This gives the maximum values of $|Z| / R_{\mathrm{dc}}$ around 1000 . This value for GMI can be achieved only in uniaxial materials with the unit direction of the anisotropy perpendicular to the conductor axis and the axial bias field, $H$, satisfying the condition in $H=H_{K}+N_{z} M_{s}+\omega^{2} / M_{s} \gamma^{2}$, where $N_{z}$ is the longitudinal demagnetizing factor and $H_{K}$ is the effective anisotropy field. Any deviation of the unit axis from the perpendicular or any fluctuation of $H_{K}$ substantially reduces the GMI effect.

\section{Materials}

The experimentally observed GMI effect in soft magnetic metals is much lower than the values predicted theoretically. Research focuses on the special heat treatments of soft magnetic metals and on the development of new materials with properties appropriate for practical GMI applications. The GMI curve, $\eta(H)$, is defined as $\eta=100 \%\left(Z(H) / Z_{0}-1\right)$, where $Z(H)$ is the impedance from a bias field, $H$, measured at a given frequency and constant driving current. $Z_{0}$ is the impedance for $H \rightarrow \infty$, which should be equal to the impedance of a nonferromagnetic conductor with both the same cross section and the same resistivity, $\rho$. Practically, $Z_{0}$ is measured at the maximum field, $H_{\max }$ available for the given experimental equipment. Some authors use $Z_{0}=Z(0)$, but that value depends on the remanent magnetic state, which may not be well defined. The parameters that characterize the GMI efficiency are the maximum GMI $\eta_{\max }$ and the maximum field sensitivity $(d \eta / d H)_{\max }$. Typical values obtained for some soft magnetic conductors are listed in Table 1.

GMI was first reported in amorphous metals, but some crystalline materials also exhibit large GMI. Sometimes the crystalline metals are even better than the amorphous ones.

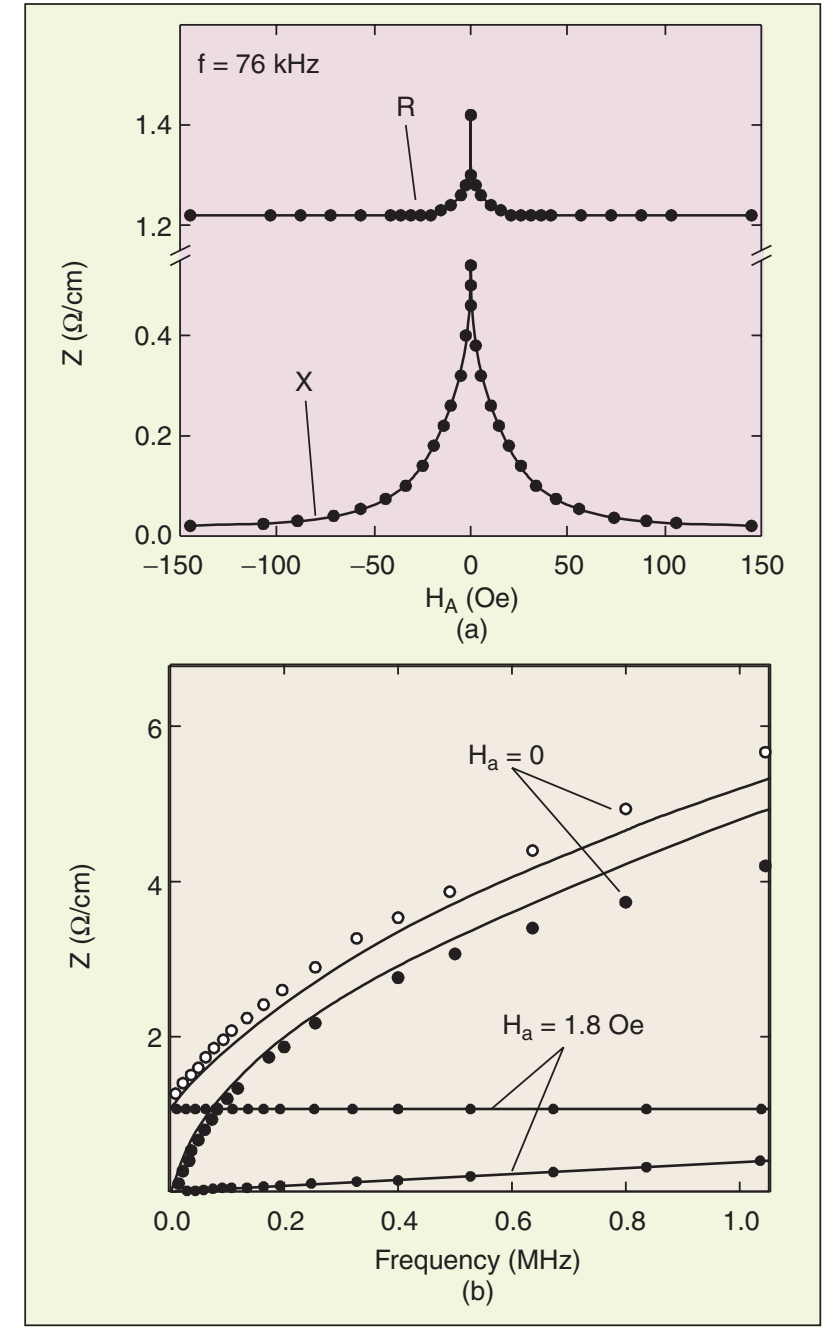

Fig. 2. GMl of an amorphous CoFeSiB wire (a) $R$, resistance, and $X$, reactance, as functions of the applied field (b) resistance (open circles) and reactance (solid circles) as functions of frequency. (Reprinted with permission from [4].)

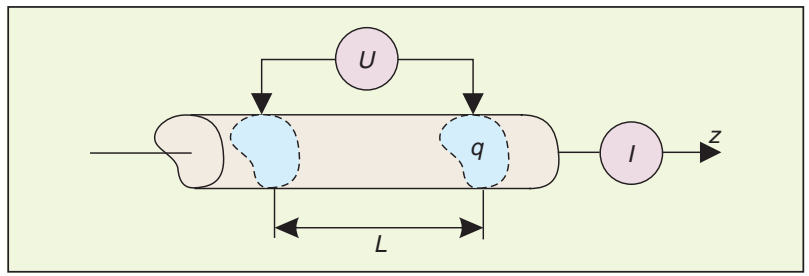

Fig. 3. Impedance definition. (Reprinted with permission from [4].)

According to theory, the largest GMI should be in materials with low resistivity, $\rho$, high saturation magnetization, $M_{s^{\prime}}$ and low damping parameter, $\alpha$. The crystalline metals have the advantage of lower resistivity, but amorphous metals have better soft magnetic behavior because they lack magnetocrystalline anisotropy. Nonmagnetostrictive materials show the best GMI performance because the magnetoelastic contribution to magnetic anisotropy substantially deteriorates the soft magnetic behavior.

Amorphous cobalt-rich ribbons, wires, and glass-covered microwires are good candidates for GMI applications. These 
Table 1. Materials for GMI Sensors (Reprinted with Permission from [4].)

\begin{tabular}{|c|c|c|c|c|}
\hline Material & Comment & $\eta_{\max }(\%)$ & $\begin{array}{l}(d \eta / d H)_{\max } \\
(\% \mathrm{~m} / \mathrm{A})\end{array}$ & $\begin{array}{l}\text { Frequency } \\
(\mathrm{MHz})\end{array}$ \\
\hline Amorphous ribbon, $\mathrm{Co}_{68.25} \mathrm{Fe}_{4.5} \mathrm{Si}_{12.25} \mathrm{~B}_{15}$ & Joule heated & 400 & - & 1 \\
\hline Amorphous wire, $\mathrm{Co}_{68.15} \mathrm{Fe}_{4.35} \mathrm{Si}_{12.5} \mathrm{~B}_{15}$ & Joule heated & 220 & 22 & 0.09 \\
\hline Amorphous microwire, $\mathrm{Co}_{68.15} \mathrm{Fe}_{4.35} \mathrm{Si}_{12.5} \mathrm{~B}_{15}$ & $\begin{array}{l}\text { Glass covered, } \\
\text { joule heated }\end{array}$ & 56 & 0.73 & 0.9 \\
\hline FINEMET wire & Annealed $600{ }^{\circ} \mathrm{C}$ & 125 & - & 4 \\
\hline Sandwich file, $\mathrm{CoSiB} / \mathrm{SiO}_{2} / \mathrm{Cu} / \mathrm{SiO}_{2} / \mathrm{COSiB}$ & $\begin{array}{l}\text { RF-sputtered in } \\
\text { magnetic field }\end{array}$ & 700 & 3.8 & 20 \\
\hline Textured Fe-3\% Si sheet & - & 360 & - & 0.1 \\
\hline $\mathrm{Ni}_{80} \mathrm{Fe}_{20}$ plated on BeCu wire & - & 530 & 4.8 & 5 \\
\hline CoP multilayers elctroplated on $\mathrm{Cu}$ wire & Twisted & 230 & - & 0.09 \\
\hline Mumetal stripe & Vacuum annealed & 310 & 0.26 & 0.6 \\
\hline
\end{tabular}

materials have the advantages of low magnetostriction and simple control of magnetic anisotropy by appropriate heat treatment; the disadvantage is high resistivity. Soft magnetic nanocrystalline metals exhibit GMI behavior similar to amorphous metals. Their somewhat higher $M_{s}$ and lower resistivity $\rho$, can lead to small improvements. The low resistivity and bulk dimensions of crystalline soft magnetic alloys lead to better performance, especially at lower driving frequencies below $1 \mathrm{MHz}$. The presence of large magnetocrystalline anisotropy (e.g., in iron-silicon alloys), however, requires a rough

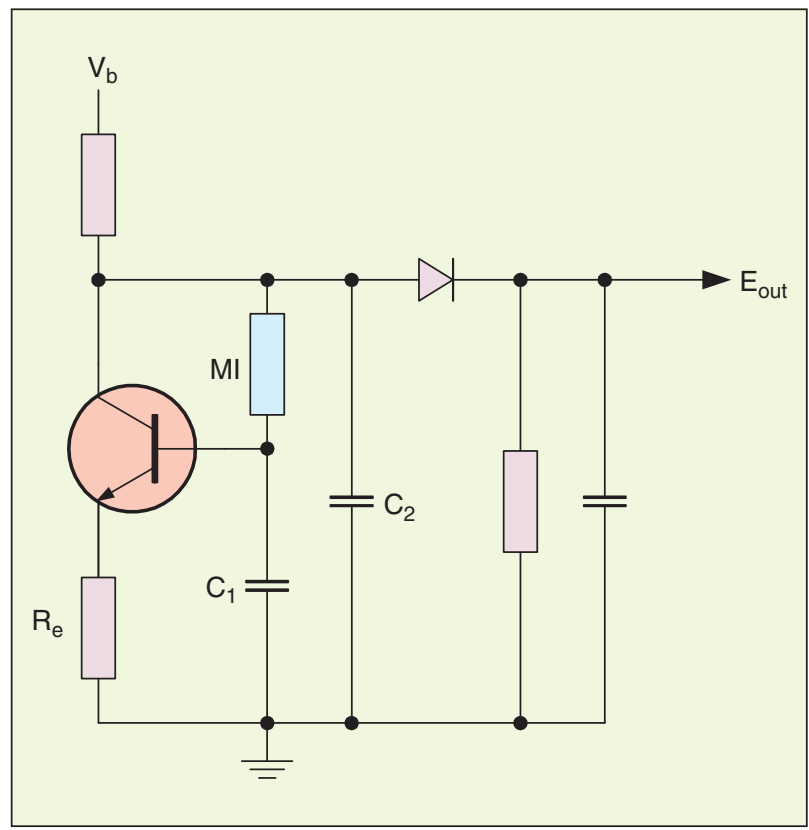

Fig. 4. Colpitts oscillator with an Ml element. (Reprinted with permission from [4].) texture of crystalline grains and proper adjustment of the driving current and the directions of the dc bias field.

Combined conductors comprising a highly conductive nonmagnetic metal core (such as $\mathrm{Cu}$ or $\mathrm{CuBe}$ ) with a thin layer of soft magnetic metal on the surface have excellent GMI behavior. An insulating interlayer between the core and the magnetic shell, in sandwich thin-film structures, results in further improvement of GMI behavior. Integrated circuits and glass-covered microwires can incorporate these thin-film structures thereby easily constructing simple sensing elements.

Sensor applications depend on the particular shape of the GMI curve, $\eta(H)$, and on both $\mu_{\text {max }}$ and the maximum field sensitivity, $(d \eta / d H)_{\max }$. Induced magnetic anisotropy and bias dc current can control the shape of a GMI curve. Wires and ribbons with transversal magnetic anisotropy have the double-peak GMI curve with the maxima close to $\pm H_{K}$. The single peak at $H=0$ is present (as in Fig. 2) if the predominate magnetic flux direction is parallel to the conductor axis. In this case, however, the $\eta_{\max }$ sharply decreases with increasing anisotropy. Helical anisotropy, which is induced in amorphous wires by torque stress or torque annealing, combined with a bias dc current, results in an asymmetric GMI curve. A linear field sensor can use this type of curve.

\section{Sensors}

Magnetic field sensors can use the high sensitivity of magnetoimpedance to external dc or low frequency ac fields where low frequency is at least ten times lower than the driving frequency. The high driving frequency has many problems like parasitic displacement currents, impedance mismatching, and the presence of reflected signals. The MI element may serve as the circuit inductance in oscillation circuits, such as the Colpitts oscillator and the resonance multivibrator, to avoid these prob- 
lems. Recently, GMI has combined with surface acoustic wave (SAW) technology in some new applications.

\section{GMI Wire in a Colpitts Oscillator}

Fig. 4 illustrates the Colpitts oscillator, which uses resonance between the inductive MI element and the capacitance values, $C_{1}$ and $C_{2}$. For oscillation frequencies around $100 \mathrm{MHz}$, the GMI signal can increase significantly. The oscillator output signal roughly follows the GMI curve; it is a nonlinear function of the applied field. A pair of MI elements used in a multivibrating oscillator circuit gives a linear field sensor [Fig. 5(b)]. The two MI elements, connected symmetrically in the multivibrator, are biased with opposite dc fields, $H_{b}$, so that in the range of applied fields $-H<H_{e x}<H_{b}$, the output voltage was approximately linear [Fig. 5(a)]. The bias field, $H_{b}$, however, requires small magnetizing solenoids to be wound around the MI elements. That complication can be avoided if dc bias current is used because the twisted wires have an asymmetrical GMI effect. The bias current in the pair of twisted MI elements flows in the opposite directions with respect to the applied dc field. Fig. 5(a) shows a linear characteristic from the applied field.

In a typical field sensor, the MI elements are parallel in the two oscillator branches and the bias fields, $H_{b}$, are opposite. A gradient field sensor uses the bias fields for the parallel elements in the same direction or the elements arranged in series with the opposite bias fields. Because the MI elements may be as short as $50 \mu \mathrm{m}$ in a $30-\mu \mathrm{m}$ wire diameter, they can detect very localized and weak magnetic fields. These types of sensors can detect biomagnetic fields and stray fields caused by cracks in steel sheets and can be used as magnetic rotary encoders of high resolution.

Miniature magnetic field sensors based on GMI effect have been used for medical applications as sensors for small movements of permanent magnets to control human physiology. They also can be used for automation and control in industry. Although GMI sensors are quite new and their development is not yet finished, their low price and high flexibility will lead to wide use in the near future.

\section{GMI and SAW}

SAW devices have been well known for more than three decades. They propagate an acoustic wave on the surface of a plain polished piezoelectric substrate. Fig. 6 illustrates a typical SAW device. A passive, wireless sensor element can use a one-port SAW device by connecting the electrical port to an antenna. The measurand determines the timing and the resonance frequency. An RF signal communicates with the sensor and information from the measurand can be recorded from the $\mathrm{RF}$ radio response signal.

Passive SAW radio sensors require no maintenance and resist severe environmental conditions such as high temperature, high electric and magnetic field strength, and even hard radiation.

Since the RF response signal interrogates the measurand and noise and interference do not disturb it, the required sig-

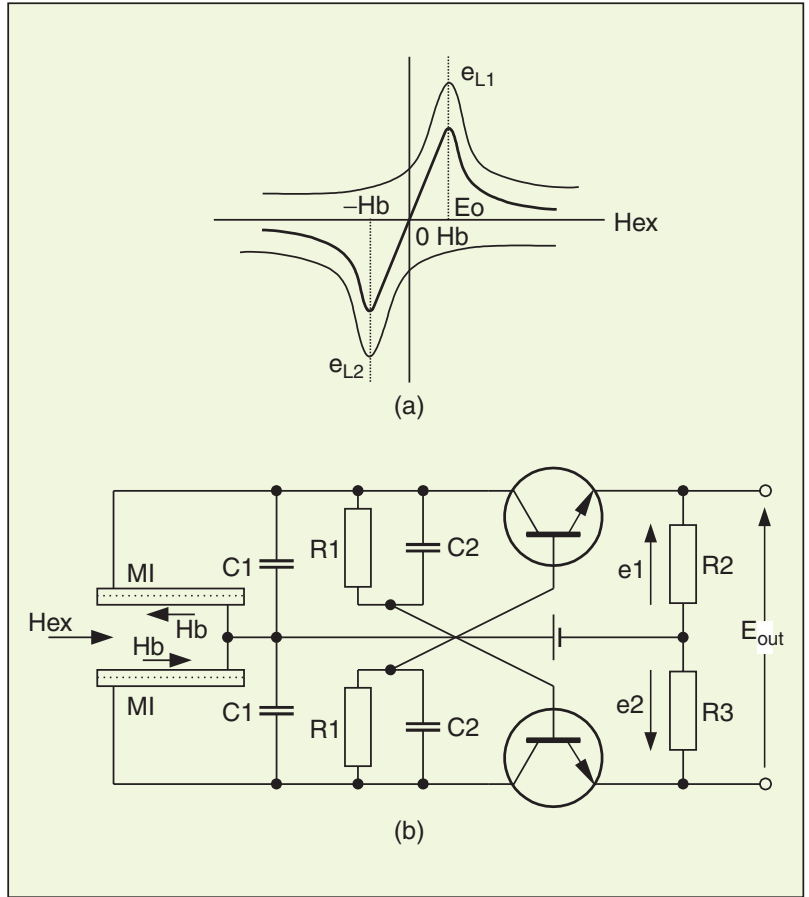

Fig. 5. Linear field sensor with multivibrator (a) field dependence of the output voltage (b) circuit design. (Reprinted with permission from [4].)

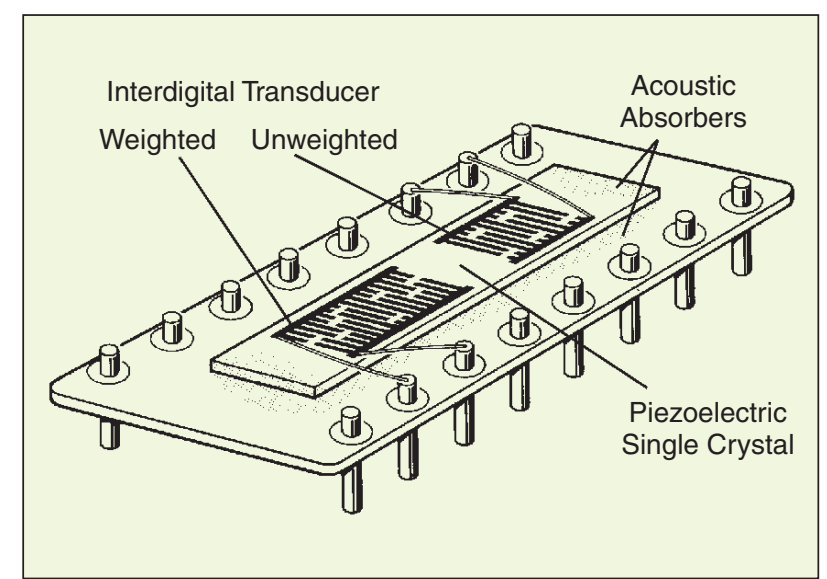

Fig. 6. SAW device.

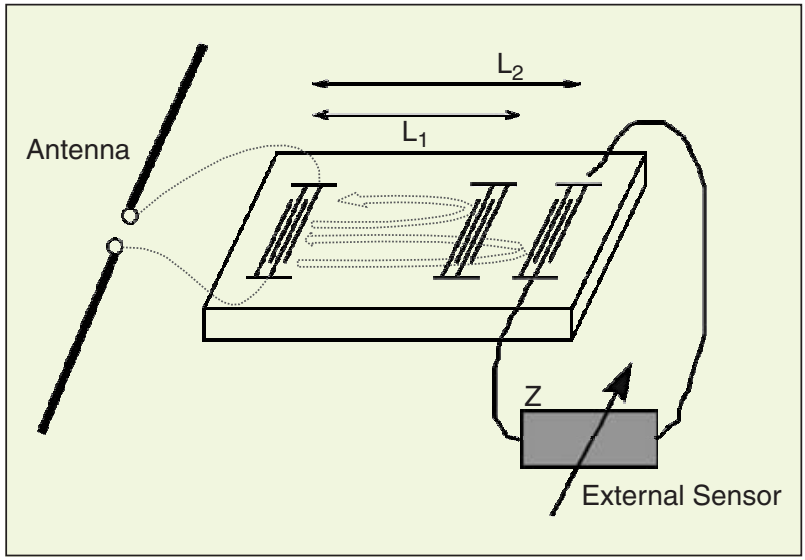

Fig. 7. Schematic layout of a two port SAW transponder with an external sensor. 


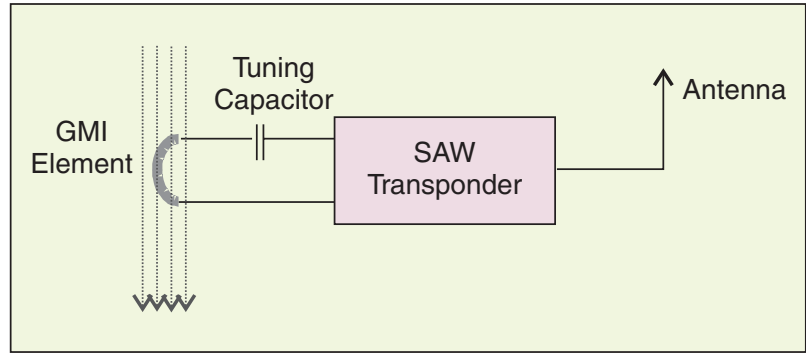

Fig. 8. Passive, wireless magnetic field sensor.

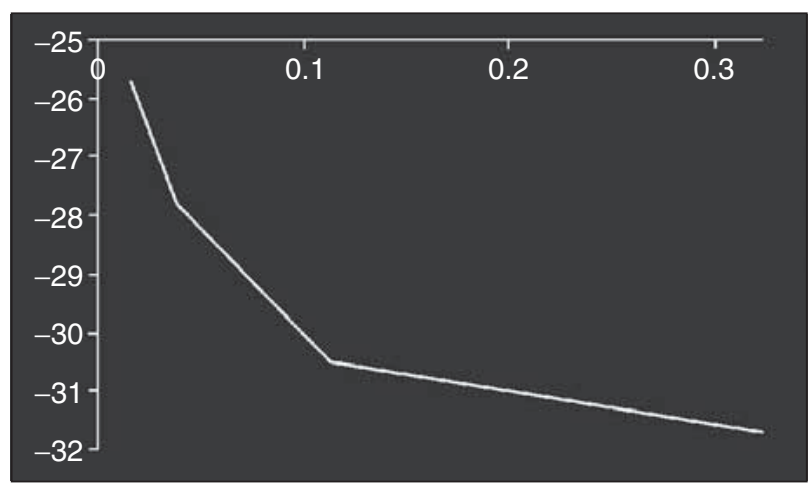

Fig. 9. Amplitude of the reflected impedance relative to the reference.

nal processing is simplified. SAW sensors represent a possible choice for applications where a wired connection to the sensor or an active radio sensor cannot be used. Since the measurand contacts the SAW substrate directly, SAW sensors are capable of remotely measuring temperature, torque, pressure, etc.

Combining GMI sensors and the SAW transponder devices produces a new wireless sensor for magnetic fields. The GMI device is coupled to the second port (interdigital transponder 2 in Fig. 7) of the SAW transponder. The circuit is adjusted to the resonance of the transducer's capacitance. Tuning the resonance for one octave in frequency by applying a magnetic field to the GMI sensor yields a sufficient effect for a radio request readout.

Fig. 8 shows a prototype sensor. Fig. 9 shows the results we obtained by measuring the amplitude of the receiving reflector relative to the reference reflector. This prototype is best suited for applications where a magnetic field has to be measured without contact and where a wired power supply is not feasible for the sensor. The sensitivity (relative signal amplitude, $B_{a}$ ) of $80 \mathrm{~dB} / \mathrm{T}$ is in the range of weak fields (up to 30 $\mathrm{mT}$ ). The development of a sensor for remote electric current measurements is in progress.

\section{Further reading}

[1] H. Hauser, R. Steindl, C. Hausleitner, A. Pohl, and J. Nicolics, "Wirelessly interrogable magnetic field sensor utilizing giant magneto-impedance effect and surface acoustic wave devices," IEEE Trans. Instrum. Meas., vol. 49, pp. 648-652, 2000.

[2] K. Mohri, K. Bushida, M. Noda, H. Yoshida, L.V. Panina, and T.Uchiyama, "Magneto-impedance element," IEEE Trans. Magn., vol. 31, pp. 2455-2457, 1992.

[3] L. V. Panina and K. Mohri, "Magneto-impedance effect in amorphous wires," Appl. Phys. Lett., vol. 65, pp. 1189-1191, 1994.

[4] P. Ripka and L. Kraus, "Magnetoimpedance and magnetoinductance" in Magnetic Sensors and Magnetometers, P. Ripka, Ed. Norwood, MA: Artech House, 2001, pp. 350-358.

Hans Hauser received his Ph.D. and the Associate Professor degree from Vienna University of Technology, Austria, in 1988 and 1994, respectively. He has been an IEEE Senior Member since 1998. His fields of interest are magnetism, dielectric materials, sensors, and actuators. Currently, he is the head of the Institute of Industrial Electronics and Material Science at the Vienna University of Technology, Austria.

Ludek Kraus was born in 1945 in Frydek-Mistek, Czech Republic. He received an M.Sc. in solid-state physics in 1968 from the Faculty of Nuclear and Technical Physics, from the Czech Technical University in Prague. In 1980, he received a Ph.D. degree in experimental physics and acoustics from the Institute of Physics, Czech Academy of Sciences. Since 1968, he has worked in the Institute of Physics, Czech Academy of Sciences in Prague. His main area of interest is the magnetism of amorphous and nanocrystalline materials.

Pavel Ripka received the CSc degree (Ph.D.) and the Docent degree from Czech Technical University in 1989 and 1996, respectively. He is a member of the steering committee of several international conferences. His fields of interest are magnetic sensors and magnetometers. He is currently a researcher and lecturer at the Department of Measurement at the Czech Technical University in Prague. 